\title{
Oxcarbazepine: A Brief Review
}

\author{
Stephanie J. Phelps, PharmD $D^{1,2,4}$ and James W. Wheless, $M D^{2,3,4}$ \\ Departments of ${ }^{1}$ Pharmacy and ${ }^{2}$ Pediatrics and ${ }^{3}$ Division of Pediatric Neurology, The University of \\ Tennessee Health Science Center and ${ }^{4}$ Le Bonheur Children's Medical Center, Memphis, Tennessee
}

Oxcarbazepine is a new anticonvulsant with an active metabolite. It is approved as mono- or adjunctive therapy for partial seizures in adults and children older than 4 years of age and as adjunctive therapy in children 2 years and older. Its spectrum of anticonvulsant activity is comparable to carbamazepine, but it has an improved pharmacokinetic profile, is better tolerated and is associated with few clinically significant drug-drug interactions.

KEYWORDS: anticonvulsant, carbamazepine, oxcarbazepine

J Pediatr Pharmacol Ther 2005;10:248-253

\section{INTRODUCTION}

Oxcarbazepine (OXC; Trileptal, Novartis Pharmaceuticals; Timox, Desitin Pharmaceuticals) is a 10-keto analog of carbamazepine (CBZ). It has the same dibenzazepine core as CBZ, but has a 5-carboxamide substitute and is different at the 10,11 position (Figure). Like CBZ it is converted to an active metabolite (10,11-dihydro-10-hydroxy-carbazepine, MHD). The Food and Drug Administration approved it as mono- or adjunctive therapy for partial seizures in adults and children older than 4 years of age and as adjunctive therapy in children 2 years and older. ${ }^{1}$ The Quality Standards Subcommittee of the American Academy of Neurology and American Epilepsy Society published evidence-based guidelines for use of the new antiepileptic drug (AED) in the treatment of children and adults with new onset ${ }^{2}$ or refractory partial and generalized epilepsies. ${ }^{3}$ The committee noted there was insufficient evidence to support OXC as monotherapy in

Address correspondence to: Stephanie J. Phelps, PharmD, 847 Monroe Avenue Suite 208D, Memphis, TN 38163, e-mail: sphelps@utmem.edu ○) 2005 Pediatric Pharmacy Advocacy Group individuals with new onset absence or primary generalized seizures. ${ }^{2}$ The committee also reported there was insufficient evidence to sup-

ABBREVIATIONS: ADH, antidiuretic hormone; AED, antiepileptic drug; CBZ, carbamazepine; MHD, 10,11dihydro-10-hydroxy-carbazepine; OXC, oxcarbazepine; UGT, UDP-glucuronyl transferase

port its use in refractory primary generalized seizures. ${ }^{3}$ Although its range of anticonvulsant activity is comparable to that of CBZ, it has an improved pharmacokinetic profile, is better tolerated and is associated with few clinically significant drug-drug interactions. ${ }^{4-7}$

\section{MECHANISM OF ACTION}

The precise mechanisms by which OXC and the MHD metabolite exert anticonvulsant effects are unknown. ${ }^{1}$ Although CBZ, OXC and MHD all block voltage-dependent sodium channels, the blockade produced by OXC/MHD occurs at a much lower concentration than that noted with CBZ in vitro. ${ }^{1,4-8} \mathrm{CBZ}$ modulates activation of the T-type calcium channels while OXC/MHD block the T-, N-, P- and R-type calcium channels. ${ }^{5,78}$ Unlike CBZ, OXC appears to have an effect on potassium channels that 


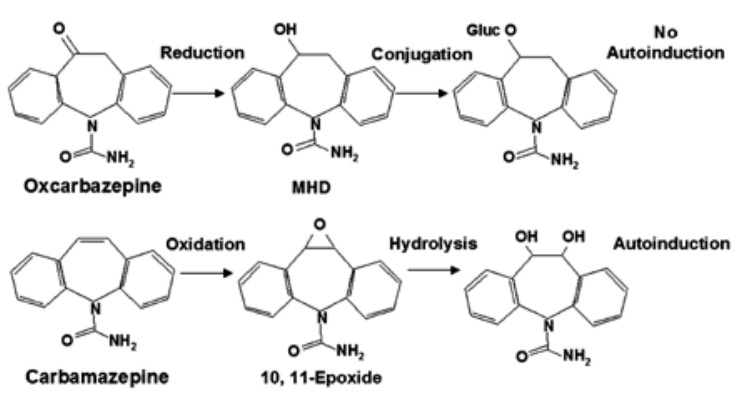

Figure. Metabolism of oxcarbamazepine and carbamazepine. MHD, monohydroxy derivative. Reprinted from reference 9, with permission from Elsevier.

contributes to anticonvulsant activity. CBZ, OXC and MHD all block the NMDA receptor. ${ }^{1,7,8}$ Neither OXC nor MHD affect binding sites for GABA or other neurotransmitter receptors and do not modulate the receptor sites. ${ }^{1,5-8}$

\section{PHARMACOKINETICS}

OXC tablets and suspension are completely absorbed and can be taken with or without food. Peak concentrations of MHD, the active metabolite, occur within 2-4 hours following a dose. ${ }^{1,7,9-12}$ Binding to serum albumin is independent of drug concentration and is about $40 \%$ for $\mathrm{MHD} ;{ }^{1,5,8}$ hence, interactions via this mechanism are clinically unimportant. Neither OXC nor MHD are bound to alpha-1-acid glycoprotein. ${ }^{1}$

OXC is rapidly and almost completely metabolized by ketoreductase (nonmicrosomal, noninducible) to MDH (Figure), which is the pharmacologically active compound.,5-9 Thus, OXC acts as a prodrug of MHD. MHD is cleared by glucuronide conjugation (UDP-glucuronyltransferase), which is sensitive to induction. Importantly, these pathways do not demonstrate genetic polymorphism. No epoxide metabolite of OXC is formed. Both OXC and MHD exhibit linear pharmacokinetics and unlike CBZ do not induce their own metabolism. ${ }^{4-7}$ The half-lives of OXC and MHD are about 2 and 9 hours, respectively. ${ }^{1}$ In children ages $2-5$ years and $6-12$ years the half-life of MHD is 4.8-6.7 hours ( \pm 1.3 hours) and 7.2-9.3 hours, respectively. ${ }^{12}$

Steady-state plasma concentrations of MHD are reached within 2-3 days following twice daily dosing. ${ }^{1}$ At steady-state the pharmacokinetics of MHD are linear and proportional to OXC doses ranging from 300 to $2400 \mathrm{mg} /$ day. ${ }^{1}$ Unlike CBZ, no dosage adjustment is required in patients with mild to moderate hepatic impairment. ${ }^{1}$

More than $95 \%$ of an OXC dose is excreted renally as glucuronides of $\mathrm{MHD}$ (49\%), unchanged MHD (27\%), and conjugates of MHD and OXC (13\%). ${ }^{1}$ Only $1 \%$ of OXC is excreted unchanged. ${ }^{1}$ There is a linear correlation between creatinine clearance and the renal clearance of MHD. The elimination half-life of MHD is prolonged to 19 hours and there is a two-fold increase in AUC in patients whose creatinine clearance is below $30 \mathrm{~mL} / \mathrm{min} .{ }^{1,7}$ For this reason, those patients should receive half the initial starting dose (i.e., $4-5 \mathrm{mg} / \mathrm{kg} /$ day), the dosing interval should be extended, and the dose should be slowly increased until the desired response in achieved. OXC dose should be decreased by $50 \%$ in patients whose creatinine clearance is $<10 \mathrm{~mL} / \mathrm{min} .{ }^{13}$

\section{DOSING}

Oxcarbazepine (Trileptal) is available as an oral tablet and suspension $(60 \mathrm{mg} / \mathrm{mL})$, which may be interchanged at equal doses (Table 1). Treatment should be initiated with $8-10 \mathrm{mg} / \mathrm{kg}$ (up to $300 \mathrm{mg} /$ day) divided twice daily., ${ }^{1,5-7}$ The dose may be increased every three days by 5 $\mathrm{mg} / \mathrm{kg} /$ day (not to exceed $600 \mathrm{mg} /$ day) until the desired response is achieved. ${ }^{1,5-7}$ In the original pediatric adjunctive therapy trial the estimated maintenance doses were based on weight; children weighing $20-29 \mathrm{~kg}$ received $900 \mathrm{mg} /$ day, those between 29.1-39 kg received $1200 \mathrm{mg} /$ day and those greater than $39 \mathrm{~kg}$ received $1800 \mathrm{mg} /$ day. ${ }^{14}$ The median dose in children is about $30 \mathrm{mg} / \mathrm{kg} /$ day; however, doses up to $70 \mathrm{mg} / \mathrm{kg} /$ day have been reported to be useful. ${ }^{12}$

Most patients in the clinical trials developed intolerable central nervous system effects at doses $\geq 2400 \mathrm{mg} /$ day. ${ }^{1}$ After a single 5 or 15 $\mathrm{mg} / \mathrm{kg}$ dose of OXC, the dose-adjusted AUC values of MHD were $30 \%-40 \%$ lower in children $<8$ years of age when compared to those older than 8 years. The mean trough serum concentrations of the MHD metabolite were also lower (34\%) in younger children when compared with older children and adults. ${ }^{12,15}$ For these reasons the mean daily dose of OXC is larger (38\%) in those below the age of 8 years. ${ }^{12}$ The clearance in children greater than 8 years old approaches 
Table 1. Comparison of dosage formulations, doses, pharmacokinetics, and reference ranges for oxcarbazepine and carbamazepine

\begin{tabular}{|c|c|c|}
\hline & OXC & CBZ \\
\hline Formulation & $\begin{array}{l}\text { tablet } \\
(150,300,600 \mathrm{mg})^{*} \\
\text { suspension } \\
(60 \mathrm{mg} / \mathrm{mL})^{*}\end{array}$ & $\begin{array}{l}\text { suspension }(100 \mathrm{mg} / 5 \mathrm{~mL}) \dagger \\
\text { tablet }(200 \mathrm{mg}) \dagger \\
\text { tablet }(100,200,400 \mathrm{mg}) \ddagger \\
\text { chewable }(100 \mathrm{mg}) \dagger \\
\text { capsule }(100,200,300 \mathrm{mg}) \S\end{array}$ \\
\hline \multicolumn{3}{|l|}{ Pediatric Dosage } \\
\hline Initial & $\begin{array}{l}8-10 \mathrm{mg} / \mathrm{kg} / \mathrm{d} \\
(300 \mathrm{mg} / \mathrm{d})\end{array}$ & $10-20 \mathrm{mg} / \mathrm{kg} / \mathrm{d}$ \\
\hline Maintenance & $\begin{array}{l}10-60 \mathrm{mg} / \mathrm{kg} / \mathrm{d} \uparrow \\
(1200-2400 \mathrm{mg} / \mathrm{d})\end{array}$ & $20-40 \mathrm{mg} / \mathrm{kg} / \mathrm{d}(1000 \mathrm{mg} / \mathrm{d})$ \\
\hline Interval & bid & bid-tid; qid (if suspension) \\
\hline \multicolumn{3}{|l|}{ Pharmacokinetics } \\
\hline $\begin{array}{l}\text { Bioavailibity } \\
\text { (time to peak, hr) }\end{array}$ & $100 \%(4-6)$ & $75-85 \%(4-8)$ \\
\hline $\mathrm{Vd}(\mathrm{L} / \mathrm{kg})$ & $0.7 \#$ & $0.8-1.6$ \\
\hline Protein Binding (\%) & $40 \#$ & $75-78$ \\
\hline Plasma half-life (hrs) & $1-3(4-12) \#$ & $12-17^{* *}$ \\
\hline Elimination & 73\% (hepatic)\# & $65 \%$ (hepatic); $15 \%$ UGT \\
\hline CYP isoenzymes & Unknown & $3 \mathrm{~A} 4,2 \mathrm{C} 8,1 \mathrm{~A} 2$ \\
\hline Reference Range & Not determined $\dagger \dagger$ & 4-12 mg/L (16-48 $\mu \mathrm{Mol} / \mathrm{L})$ \\
\hline
\end{tabular}

CBZ, carbamazepine; OXC, oxcarbazepine; UGT, uridine diphosphate glucuronosyltransferase

* Trileptal (Novartis Pharmaceuticals, Hanover, NJ) and Timox (Desitin, Hamburg Germany)

+ Tegretol and generic

$\neq$ Tegretol $X R$

$\S$ Carbatrol

I $20-29 \mathrm{~kg}=900 \mathrm{mg} /$ day; $29.1-39 \mathrm{~kg}=1200 \mathrm{mg} /$ day $>39 \mathrm{~kg}=1800 \mathrm{mg} /$ day

\# values for monohydroxy (MHD) metabolite

** on repeat dosing (10-14 hrs in children 10-13 years of age)

t+ Some suggest a reference range of $15-35 \mathrm{mg} / \mathrm{L}$, however, the correlation between these concentrations with efficacy and toxicity have not been proven

that of adults.

Patients receiving other anticonvulsants may be converted to monotherapy with OXC. When OXC is substituted for CBZ, the replacement dose should be 1.2 to 1.5 times the CBZ dose. ${ }^{1,6}$ The lower conversion factor should be used in the elderly and in patients receiving large doses of CBZ. If a rapid substitution is desired, the CBZ should be held and full dose OXC should be initiated with the next scheduled dose. Individuals receiving other anticonvulsants or desiring a slower substitution should be started on OXC while concurrently reducing the dose of the other medication(s). ${ }^{1,6}$ The concomitant anticonvulsant should be completely withdrawn over 3-6 weeks, and the maximum dose of OXC should be reached in about 2-4 weeks. ${ }^{1,6}$ In patients receiving CBZ (or other enzyme-inducing AEDs), substitution with oxcarbazepine can result in deinduction of hepatic enzyme systems with subsequent increase in plasma concentrations of the remaining AEDs. Dosage adjustment may be needed in patients who are switched to oxcarbazepine from other AEDs with enzyme-inducing properties.

\section{TERATOGENICITY AND EXCRETION IN BREAST MILK}

OXC and MHD are not metabolized by the placenta but do significantly cross the placenta. ${ }^{1,15}$ The published literature is limited and 
does not allow one to compare OXC to the other anticonvulsants; however, major congenital anomalies have not been noted. ${ }^{15}$ Because the offspring of animals treated with the maximum recommended human dose of OXC or MHD during pregnancy developed fetal structural abnormalities and other manifestations of developmental toxicity, the FDA classified OXC as a Category $\mathrm{C}$ (compared to $\mathrm{CBZ}$ which is a Category D). ${ }^{1}$ Montouris reviewed the literature from pregnancies involving maternal exposure to OXC monotherapy $(\mathrm{n}=248)$ and adjunctive therapy $(\mathrm{n}=61) .{ }^{16}$ The author noted that the relatively small number of women limited definitive conclusions, but OXC monotherapy did not appear to increase the rate of malformations compared to the rate in the general (non-epileptic) population. Worldwide collaborative pregnancy registries are now being used to better define the likelihood of anticonvulsant-induced teratogenicity. Until more information is available, the benefit to the mother should be weighed against the risk to the fetus. OXC is excreted in breast milk and the risk to infants should be considered. ${ }^{1}$

\section{SIDE EFFECTS}

The most frequent side effects involve the CNS and include headache, somnolence, dizziness, and nausea, all of which resolve following dosage adjustment. ${ }^{1,17}$ OXC has been associated with CNS related adverse events such as psychomotor slowing, concentration difficulties, and speech or language problems. ${ }^{1}$

Rash is the most common idiosyncratic reaction reported with OXC (3\%), but the occurrence is less than that noted with carbamazepine (5\%). ${ }^{1,6}$ Benign, maculopapular, urticarial, and morbilliform rash occurs between the first and second week of therapy and is generally managed with symptomatic measures (e.g., diphenhydramine) and discontinuation of OXC. Although rare, exfoliative dermatitis and Stevens-Johnson syndrome may also occur. Cross-reactivity exists between OXC and CBZ. Allergic skin reactions due to OXC may occur in a third of adults who developed a rash on CBZ. ${ }^{5}$ No data on percent cross-reactivity exist in children. Beran reported $100 \%$ cross-reactivity in three patients who had a serious skin reaction to CBZ (e.g., exfoliative dermatitis). ${ }^{18}$
Although rare, multiorgan hypersensitivity disorders characterized by rash, fever, lymphadenopathy, abnormal liver function tests, eosinophilia and arthralgia have been reported for CBZ and OXC. Patients who experience a moderate to severe dermatological reaction generally should avoid lamotrigine or other aromatic AEDs including OXC.

Asymptomatic hyponatremia (serum sodium $<125 \mathrm{mEq} / \mathrm{L}$ ) has been noted in about $3 \%$ of patients given OXC and occurs more often than with CBZ. ${ }^{6}$ Risk factors include increasing age, dose (> $30 \mathrm{mg} / \mathrm{kg} /$ day) and concomitant administration of diuretics. ${ }^{7}$ Hyponatremia occurs rarely in those $<18$ years of age. ${ }^{6}$ While both OXC and CBZ cause hyponatremia and water retention, these may occur via different mechanisms. CBZ probably increases antidiuretic hormone $(\mathrm{ADH})$ secretion, but the mechanism of OXC-induced hyponatremia is not related to an increase in $\mathrm{ADH}$. It is thought to be due to a direct effect of OXC on renal collecting tubules, or to an exaggerated response to circulating $\mathrm{ADH} .{ }^{19}$ Although there have been reports of patients who developed hyponatremia more than 1 year after initiation of therapy, it generally occurs during the first three months of treatment. ${ }^{1}$

Clinical manifestations of hyponatremia are similar to those seen in water intoxication and depend on rate of change in sodium concentration more than the absolute value. At low serum sodium concentrations $(<120$ $\mathrm{mEq} / \mathrm{L}$ ), patients may report headache, confusion, dizziness, restlessness, irritability, muscle cramps, confusion, or loss of seizure control. Even in asymptomatic individuals, sodium < $120 \mathrm{mEq} / \mathrm{L}$ should be treated because rapid clinical deterioration may occur.

Serum sodium generally normalizes within a few days after OXC dose reduction or discontinuation. ${ }^{1}$ If additional intervention is needed the patient may be fluid restricted. Demeclocycline has been used in those who require continued CBZ, but there are no reports of its use in OXC-induced hyponatremia. Serum sodium should be measured if the patient is receiving other medications known to cause inappropriate $\mathrm{ADH}$ secretion or that affect sodium balance, or when symptoms of hyponatremia develop. Although CBZ can cause cardiac conduction disturbances, OXC is free 
Table 2. Effect of oxcarbazepine (OXC) and its monohydroxy metabolite MHD on other anticonvulsants $s^{4,5,7,10,20,21}$

\begin{tabular}{|c|c|c|}
\hline AED & $\begin{array}{c}\text { OXC on } \\
\text { other } \\
\text { AEDs }\end{array}$ & $\begin{array}{l}\text { AEDs on } \\
\text { MHD }\end{array}$ \\
\hline Carbamazepine & $\downarrow(0-22 \%)$ & $\downarrow(40 \%)$ \\
\hline $\begin{array}{l}\text { Carbamazepine 10, } \\
\text { 11-epoxide }\end{array}$ & $\uparrow(30 \%)$ & NA \\
\hline Felbamate & NA & $\leftrightarrow$ \\
\hline Gabapentin & $\leftrightarrow$ & $\leftrightarrow$ \\
\hline Lamotrigine & $\downarrow(0-30 \%)$ & $\leftrightarrow$ \\
\hline Levetiracetam & $\downarrow(0-20 \%)$ & NA \\
\hline Phenobarbital & $\uparrow(15 \%)$ & $\downarrow(31 \%)$ \\
\hline Phenytoin & $\uparrow(0-40 \%)$ & $\downarrow(30-35 \%)$ \\
\hline Topiramate & $\downarrow$ & NA \\
\hline Valproic acid & $\leftrightarrow$ & $\downarrow(0-18 \%)$ \\
\hline
\end{tabular}

AED, antiepileptic drug; MHD, 10,11-dihydro-10-hydroxy-carbazepine; NA, not available

of cardiac toxicity. Likewise, no clinically important hematological, renal or liver changes have been reported.

\section{DRUG INTERACTIONS}

While CBZ is associated with numerous clinically significant drug-drug interactions, OXC has a low potential for drug interactions. ${ }^{20,21}$ The ability of OXC and MHD to inhibit select cytochrome $\mathrm{P} 450$ isozymes was assessed using human liver microsomes. Neither OXC nor MHD inhibited CYP1A2, CYP2A6, CYP2C9, CYP2D6, CYP2E1, CYP4A9 or CYP4A11. ${ }^{1}$ MHD is a weak inducer of UDP-glucuronyl transferase (UGT) and is unlikely to affect drugs (e.g., valproic acid, lamotrigine) that are mainly eliminated by via this pathway. OXC/MHD can inhibit CYP2C19 resulting in elevated serum phenytoin concentrations or signs of phenytoin toxicity and induce CYP3A4 and CYP3A5 thereby interfering with the metabolism of some medications (Table 2). 6,7,10,11 These effects occur at high serum concentrations; hence, their activity on CYP3A4/5 should not be clinically important. Because plasma protein binding of MHD is low (40\%), the likelihood of interactions based on competition for protein binding sites is not likely. Importantly, several anticonvulsants (i.e., carbamazepine, phenytoin and phenobarbital) that induce cy- tochrome P450 isozymes can decrease serum concentrations of MHD. ${ }^{6,7,10,11}$

Concomitant administration of OXC decreases serum concentrations of two hormonal components (ethinylestradiol and levonorgestrel) found in oral contraceptives. ${ }^{22,23}$ Women of childbearing age should be advised that OXC may cause this method of contraception to be less effective and that non-hormonal forms of contraception should be used. If oral contraceptives are used, a high-dosage estrogen contraceptive should be prescribed and the patient should be monitored for signs of reduced hormonal cover. OXC decreased the AUC of felodipine by $28 \%$, and verapamil caused a $20 \%$ decrease in serum concentrations of MHD. Cimetidine, erythromycin, and dextropropoxyphene do not affect MHD. Likewise, there is no evidence of an interaction with warfarin.

\section{MONITORING}

The "therapeutic" serum concentration range for an anticonvulsant can be useful in individualizing therapy. Within this range, patients may achieve seizure control without experiencing unacceptable side effects. Although this range can serve as a useful guide to therapy, the clinician should strive to determine the optimum serum concentration for each patient rather than relying on published ranges. ${ }^{24}$ While a "therapeutic" range is available for $\mathrm{MDH}$ (15$35 \mathrm{mg} / \mathrm{L} ; 50-140 \mu \mathrm{mol} / \mathrm{L}),{ }^{25}$ this range reflects concentrations achieved during clinical trials and does not necessarily correspond with efficacy or toxicity. Assays for oxcarbazepine are generally not available in clinical laboratories; hence, it may require days to obtain results. The company recommends that plasma concentrations of the concomitant AEDs be monitored during the period of OXC titration, as these serum values may be altered, especially at OXC doses greater than $1200 \mathrm{mg} /$ day. $^{1}$

DISCLOSURE: Dr. Phelps declares no conflicts or financial interest in any product or service mentioned in the manuscript, including grants, equipment, medications, employment, gifts, and honoraria. Dr. Wheless has grant support from the National Institutes of Health, Abbott Laboratories, King Pharmaceuticals, Ortho-McNeil, Ovation and UCB Pharma. He is on the Speaker's Bureau for Cyberonics, GlaxoSmithKline, Novartis, Ortho-McNeil, Pfizer, UCB Pharma, and Valeant Pharmaceuticals. Dr. Wheless is also 
a consultant to Abbott Pharmaceuticals, Cyberonics, King Pharmaceuticals, Marinus, Novartis, Questor, Ovations, UCB Pharma, and Valeant Pharmaceuticals.

\section{REFERENCES}

1. Physicians' desk reference. Thompson PDR, Montvale, NJ. Version 7.0.310a, 2005.

2. French JA, Kanner AM, Bautista J, et al. Efficacy and tolerability of the new antiepileptic drugs I: treatment of new onset epilepsy: report of the Therapeutics and Technology Assessment Subcommittee and Quality Standards Subcommittee of the American Academy of Neurology and the American Epilepsy Society. Neurology 2004;62:1252-1260.

3. French JA, Kanner AM, Bautista J, et al. Efficacy and tolerability of the new antiepileptic drugs II: treatment of refractory epilepsy: report of the Therapeutics and Technology Assessment Subcommittee and Quality Standards Subcommittee of the American Academy of Neurology and the American Epilepsy Society. Neurology 2004;62:1261-1273.

4. LaRoche SM, Helmers SL. The new antiepileptic drugs: clinical applications. JAMA 2004;291:615-620.

5. Glauser TA. Oxcarbazepine in the treatment of epilepsy. Pharmacotherapy 2001;21:904-919.

6. Schmidt D, Sachdeo R. Oxcarbazepine for treatment of partial epilepsy: A review and recommendations for clinical use. Epilepsy Behav 2000;1:396-405.

7. Schmidt D, Elger CE. What is the evidence that oxcarbazepine and carbamazepine are distinctly different antiepileptic drugs? Epilepsy Behav 2004;5:627-635.

8. LaRoche SM, Helmers SL. The new antiepileptic drugs: scientific review. JAMA 2004;291:605-614.

9. Lloyd P, Flesch G, Dieterle W. Clinical pharmacology and pharmacokinetics of oxcarbazepine. Epilepsia 1994;35 Suppl 3: S10-13.

10. May TW, Korn-Merker E, Rambeck B. Clinical pharmacokinetics of oxcarbazepine. Clin Pharmacokinet 2003;42:1023-1042.

11. Bang LM, Goa KL. Spotlight on oxcarbazepine in epilepsy. CNS Drugs 2004;18:57-61.
12. Rey E, Bulteau C, Motte J, et al. Oxcarbazepine pharmacokinetics and tolerability in children with inadequately controlled epilepsy. J Clin Pharmacol 2004;44:12901300.

13. Rouan MC, Lecaillon JB, Godbillon J, et al. The effect of renal impairment on the pharmacokinetics of oxcarbazepine and its metabolites. Eur J Clin Pharmacol 1994;47:161-167.

14. Glauser TA, Nigro M, Sachdeo R, et al. Adjunctive therapy with carbamazepine in children with partial seizures. Neurology 2000;54:2237-2244.

15. Perucca E. Birth defects after prenatal exposure to antiepileptic drugs. Lancet Neurol 2005;4:781-786.

16. Montouris G. Safety of the newer antiepileptic drug oxcarbazepine during pregnancy. Curr Med Res Opin 2005;21:693-701.

17. Bourgeois BF, D’Souza J. Long-term safety and tolerability of oxcarbazepine in children: a review of clinical experience. Epilepsy Behav 2005;7:375-382. Epub 2005 Sep 19.

18. Beran RG. Cross-reactive skin eruption with both carbamazepine and oxcarbazepine. Epilepsia 1993;34:163-165.

19. Sachdeo RC, Wasserstein A, Mesenbrink PJ, D'Souza J. Effects of oxcarbazepine on sodium concentration and water handling. Ann Neurol 2002;51:613-620.

20. Sallas WM, Milosavljev S, D'souza J, Hossain M. Pharmacokinetic drug interactions in children taking oxcarbazepine. Clin Pharmacol Ther 2003;74:138-149.

21. Hulsman JA, Rentmeester TW, Banfield $\mathrm{CR}$, et al. Effect of felbamate on the pharmacokinetics of the monohydroxy and dihydroxy metabolite of oxcarbazepine. Clin Pharmacol Ther 1995;58:383-389.

22. Fattore C, Cipolla G, Gatti G, et al. Induction of ethinylestradiol and levonorgestrel metabolism by oxcarbazepine in healthy women. Epilepsia 1999;40:783-787.

23. Crawford P. Interactions between antiepileptic drugs and hormonal contraception. CNS Drugs 2002;16:263-272.

24. Dodson WE. Level off. Neurology 1989;39:1009-1010.

25. Johannessen SI. Can pharmacokinetic variability be controlled for the patient's benefit? Ther Drug Monit 2005;27:710-713. 